\title{
Evaluation of nutritional therapy in paediatric burn injuries
}

\author{
Jakub Noskiewicz'1, Paweł Juszczak², Przemysław Mańkowski \\ 'Department of Paediatric Surgery, Traumatology, and Urology, Poznan University of Medical Sciences, Poznan, Poland \\ 2Department of Human Nutrition and Hygiene, Poznan University of Life Sciences, Poznan, Poland
}

\section{ABSTRACT}

\begin{abstract}
Aim of the study: Burn injuries occur mainly in children, primarily between two and four years of age. The most common cause of burns is contact with hot liquid. Apart from local treatment of damaged tissues, an important role in burn injury management is played by immediate implementation of burn disease management. This procedure, aimed at mitigation of systemic effects of thermal injury, consists of an adequate nutritional procedure, including supply of high-protein diet. The purpose of this study is to evaluate the nutritional procedure in children with burns.

Material and methods: An evaluation was performed on nutritional interventions implemented in children hospitalised for burn injuries in 2010-2017. The effectiveness of the use of oral nutritional supplements was researched in detail.

Results: In the studied period, 310 children were hospitalised for burns. Implementation of temporary parenteral nutrition was necessary in $1.94 \%$ of patients. In the subsequent years of the study, a significant increase in the supply of oral nutritional supplements (ONS) within the nutritional intervention was observed $(2.6 \%$ vs. $45.7 \%$ ). The applied nutritional interventions enabled maintenance of a proper nutritional status during treatment at the surgery department. The values of total protein and albumins as of the last day of hospitalisation displayed no significant differences compared to the values determined at admission to hospital. Paediatric patients administered with ONS had significantly higher total protein values at discharge from hospital ( $6.87 \pm 0.12 \mathrm{~g} / \mathrm{dl}$ for ONS vs. $6.26 \pm 0.24 \mathrm{~g} / \mathrm{dl}$ for N-ONS) and displayed a higher rate of burn wound healing $(p=0.030)$.

Conclusions: Implementation of adequate nutritional intervention affects both the course of burn disease management and the wound healing rate. Oral nutritional supplements play an increasing role in the treatment of paediatric patients with burn injuries. Administration of ONS has a significant impact on the nutritional status of burnt children and accelerates the wound healing process.
\end{abstract}

\section{KEY WORDS:}

burn, child, nutritional therapy, oral nutritional supplements.

\section{INTRODUCTION}

Burn injuries occur mainly in children, primarily in patients up to the third year of life. Apart from local treatment of damaged tissues, an important role in burn injury management is played by immediate implementation of burn disease management. This procedure, aimed at mitigation of systemic effects of thermal injury, consists of an adequate nutritional procedure. Apart from pain and shock therapy, it plays a key role in treatment of patients with burn injuries and significantly affects the recovery process and wound healing rate.

\section{ADDRESS FOR CORRESPONDENCE:}

Jakub Noskiewicz, Department of Paediatric Surgery, Traumatology, and Urology, Poznan University of Medical Sciences, 27/33 Szpitalna St., 60-572 Poznan, Poland, ORCID: 0000-0001-5473-0824,

e-mail: jnoskiewicz@op.pl 
Thermal injury always induces a hypermetabolic response. By catabolic processes and loss of fat-free body weight, it promotes pro-inflammatory responses as well as immunological and endocrine disorders. Therefore, implementation of an adequate nutritional intervention at the initial stage of treatment significantly affects its course and further rehabilitation. It also reduces the risk of early or late complications, therapy length, and probability of death. The purpose of the study is to evaluate the nutritional procedure in children with burn injuries, in particular the need to implement appropriate nutritional intervention - especially with the use of oral nutritional supplements (ONS).

\section{MATERIAL AND METHODS}

An evaluation was performed of nutritional interventions implemented in children hospitalised for burn injuries in the Department of Paediatric Surgery, Traumatology, and Urology of the Poznan University of Medical Sciences in 2010-2017. The analyses used, among others, detailed evaluation records of the patients with thermal injuries, considering the applied nutritional procedure. All patients (up to 18 years of age) treated for isolated thermal injuries were enrolled in the study. None of the patients had absorption disorders. No exclusion criteria were applied.

The analysed population consisted of 310 children, including $66.8 \%$ of boys (207 patients) and $33.2 \%$ of girls (103 patients). The median age of patients was one year. The vast majority of them $(72.9 \%)$ were patients up to three years of age, but only $1.2 \%$ of children (four patients) were under the age of one year. The main cause of burns was contact with hot liquid, most frequently in the area of: upper limbs, chest, and head and neck. All wounds demonstrated a mosaic nature with dominating second-degree burns (84.8\%). On average, approximately $10.0 \%$ of body surface was injured. Nine per cent (28) of children with burns required surgery. In most cases, the procedure consisted of necrectomy and autologous split-thickness skin graft transfer.

The patients were evaluated at admission to hospital and during treatment at the surgery department, with

TABLE 1. The main characteristics of a hospital diet for children with burn injuries

Easily digestible diet with increased protein supplementation
( $3 \mathrm{~g}$ protein/ $\mathrm{kg}$ b.w./24 $\mathrm{h}$ ).
The protein covers daily energy needs in $10-15 \%$.
$2 / 3$ of the protein should be a wholesome protein and come
from animal products.
Increased energy supply in the diet (to use protein for building
and regeneration purposes).
Carbohydrate covers daily energy needs in $50-55 \%$.
Fat covers daily energy needs in $30-35 \%$.
Salt is limited in the diet.

a view to nutrition in the context of necessary implementation of an adequate nutritional intervention. The evaluation was performed by a surgeon in collaboration with a dietician, considering the current standards of the World Health Organisation (WHO) and using the laboratory parameters of nutritional status assessment (e.g. total protein, albumins). The caloric need was determined empirically by multiplying the basal metabolic rate (calculated using the Harris-Benedict formula) by the conversion factors $(1+[2 \times$ percentage of burned body surface $])$. The caloric intake of children was assessed empirically. It was based on registered information on the amount and type of meals eaten by the patient. The type of introduced nutritional intervention and its effectiveness were also evaluated. The focus was on administration of ONS in burn management. In the second phase of the study, the group of patients administered with ONS within the nutritional intervention at the surgery department were compared with the corresponding population of patients who were not administered with such formulas. The general condition of children after hospitalisation in both groups was compared, with a particular focus on nutritional status parameters and wound healing assessment. The acquired data were analysed using the descriptive statistics, methods, and tools, adopting the significance level of $p<0.05$. Consent to perform the studies was issued by the Bioethical Committee at Poznan University of Medical Sciences (no. 175/17).

\section{RESULTS}

The main factors modifying the energy intake included burn injury extensiveness (surface) and the need for implementation of surgical procedures (wound surgery with general anaesthesia, split-thickness graft). All patients above one year of age were put on a high-energy diet rich in proteins (Table 1), while the infants were fed mostly with their mother's milk. Only $1.3 \%$ of children were breastfed. Among those patients, no high-protein or rich-energy supplementation was used. Mother's milk completely met the energy needs of the burned infants. Despite implementation of an adequate nutritional procedure dedicated to thermal injuries, all patients under study required additional nutrition with food products purchased by the parents. $1.9 \%$ of patients required implementation of temporary parenteral nutrition, which resulted primarily from their severe general condition, advanced progression of burn disease, and their stay at the paediatric intensive care unit. Parenteral nutrition formulas were prepared each time by the hospital pharmacy with consideration to the individual nutritional and metabolic needs of treated paediatric patients. $45.7 \%$ of children required food interventions in 2017. The majority of them (93.8\%) were administered only orally or enterally, also using ONS.

The population under study demonstrated proper nutritional status at the time of thermal injury. No low body 
TABLE 2. The comparison of the nutritional status of burned children at the beginning and end of hospitalisation (percentages or mean and standard deviation)

\begin{tabular}{|l|c|c|c|}
\hline & $\begin{array}{c}\text { Admission } \\
\text { to the hospital }\end{array}$ & $\begin{array}{c}\text { Discharge from } \\
\text { the hospital }\end{array}$ & $p$-value \\
\hline Correct nutritional status (\%) & 100 & 100 & $>0.999$ \\
\hline Total protein (mean) & $6.20 \pm 0.81 \mathrm{~g} / \mathrm{dl}$ & $6.13 \pm 0.88 \mathrm{~g} / \mathrm{dl}$ & 0.794 \\
\hline Albumin (mean) & $4.22 \pm 0.47 \mathrm{~g} / \mathrm{dl}$ & $4.26 \pm 0.28 \mathrm{~g} / \mathrm{dl}$ & 0.323 \\
\hline
\end{tabular}

weight or short stature were observed. The values of total protein $(6.46 \pm 0.55 \mathrm{~g} / \mathrm{dl})$ and albumins $(4.22 \pm 0.44 \mathrm{~g} / \mathrm{dl})$ fell within the normal range. The applied nutritional interventions enabled maintenance of a proper nutritional status during treatment at the surgery department. The values of total protein and albumins as of the last day of hospitalisation displayed no significant differences compared to the values determined at admission to hospital (Table 2). With regard to high body weight variability resulting from significant body fluid loss and intensive fluid therapy during treatment, the measurement of this parameter was inadequate for nutritional status assessment. No disturbances of glycaemia and hyperglycaemic states were observed in the studied population.

A significant growth of administration of ONS within the nutritional intervention in children with burn injuries was observed. This growth was exponential - from $2.6 \%$ of patients in the first years to $45.7 \%$ of patients in 2017 (Fig. 1). The main criterion for including ONS was inadequate energy supply by the hospital diet. ONS constituted a significant supplementation to the hospital high-energy diet rich in proteins applied in patients with burn wounds. This type of nutritional management was successfully implemented also in younger patients after the first year of age. In the last years of the study, ONS was administered to all children with insufficient energy or protein supply despite introduction of an adequate hospital diet (Table 3). In most cases, supply of a single serving of ONS daily was sufficient (average volume of $200 \mathrm{ml}$, average energy supply of $1.5 \mathrm{kcal} / \mathrm{ml}$ ). Maximum dose was $600 \mathrm{ml} / 24 \mathrm{~h}$ of ONS during nutritional intervention in a teenager treated for full-thickness burn (third degree). Flavour was the main selection criterion for a given formula and tolerance indicator in a given population. The best tolerance was recorded for chocolate flavour.

The population of patients administered with ONS were compared with a group of children not administered with ONS (N-ONS). These groups were identical in terms of: age (median: 1.5 years for ONS and for N-ONS), sex (62.5\% of boys for ONS and 73.9\% of boys for N-ONS), nutrition at admission to hospital as well as burned surface (averages of $6.4 \%$ for ONS and $8.3 \%$ for N-ONS), and the degree (median: 2A grade for ONS and for N-ONS) and scheme of thermal injury. The burn wound treatment method was also the same. $12.5 \%$ of children from the ONS group required an autologous split-thickness skin graft transfer, while all patients in the N-ONS group

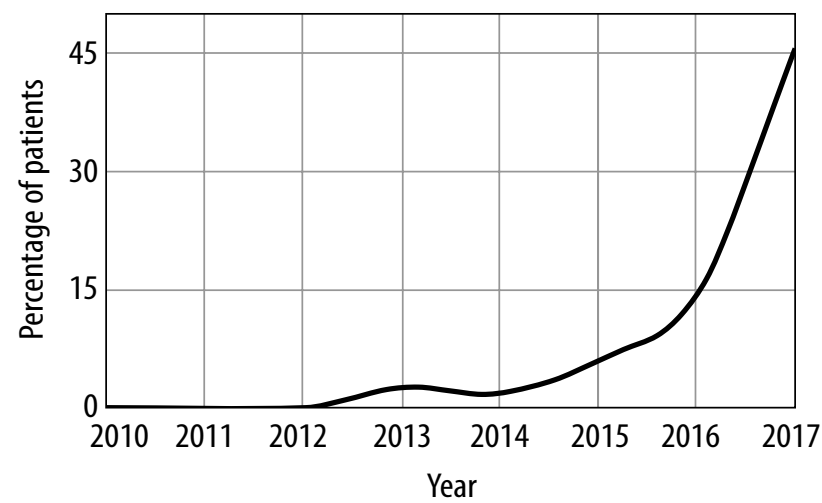

FIGURE 1. Frequency of use of oral nutritional supplements as part of nutritional intervention in 2010-2017

TABLE 3. The composition of oral nutritional supplements used in children with burn injuries

\begin{tabular}{|c|c|c|c|}
\hline \multicolumn{2}{|l|}{ Component } & Unit & Per $100 \mathrm{ml}$ \\
\hline \multicolumn{2}{|l|}{ Energy } & kcal & 153.00 \\
\hline \multicolumn{2}{|l|}{ Protein } & g & 3.30 \\
\hline \multicolumn{2}{|l|}{ Carbohydrate } & g & 18.80 \\
\hline \multicolumn{2}{|l|}{ Sugars } & g & 7.40 \\
\hline \multicolumn{2}{|l|}{ Fat } & $g$ & 6.80 \\
\hline \multicolumn{2}{|l|}{ Dietary fibre } & g & 1.50 \\
\hline \multicolumn{2}{|l|}{ Salt } & $\mathrm{g}$ & 0.17 \\
\hline \multicolumn{4}{|c|}{ Energy distribution (\%) } \\
\hline Protein & Carbohydrate & Fat & Dietary fibre \\
\hline 9 & 49 & 40 & 2 \\
\hline
\end{tabular}

were treated conservatively. The lengths of hospitalisation in both populations under study were comparable $(p=0.099)$. The children administered with ONS in most cases left the hospital with completely healed thermal injuries, while the burn wounds of the patients not administered with ONS were primarily at the stage of partial epithelialisation ( $p=0.030)$ at discharge from hospital. There were significant differences $(p=0.026)$ in the values of total protein determined at discharge from hospital (averages of $6.87 \pm 0.12 \mathrm{~g} / \mathrm{dl}$ for ONS and $6.26 \pm 0.24 \mathrm{~g} / \mathrm{dl}$ for N-ONS) between both groups, although these parameters did not deviate from the laboratory standards. No difference in the values of albumins $(p=0.106)$, haematocrit $(p=0.468)$, and haemoglobin $(p=0.598)$ deter- 
TABLE 4. The comparison of burned children receiving and not receiving oral nutritional supplements - laboratory parameters at discharge from the hospital (mean and standard deviation)

\begin{tabular}{|l|c|c|c|}
\hline & ONS & N-ONS & $p$-value \\
\hline Total protein & $6.87 \pm 0.24 \mathrm{~g} / \mathrm{dl}$ & $6.26 \pm 0.12 \mathrm{~g} / \mathrm{dl}$ & 0.026 \\
\hline Albumin & $4.22 \pm 0.47 \mathrm{~g} / \mathrm{dl}$ & $4.26 \pm 0.28 \mathrm{~g} / \mathrm{dl}$ & 0.106 \\
\hline Haemoglobin & $11.8 \pm 1.0 \mathrm{~g} / \mathrm{dl}$ & $11.6 \pm 0.9 \mathrm{~g} / \mathrm{dl}$ & 0.580 \\
\hline Haematocrit & $35.0 \pm 2.7 \%$ & $34.1 \pm 3.1 \%$ & 0.468 \\
\hline
\end{tabular}

ONS - patients receiving oral nutritional supplements, $\mathrm{N}$-ONS - patients not receiving oral nutritional supplements

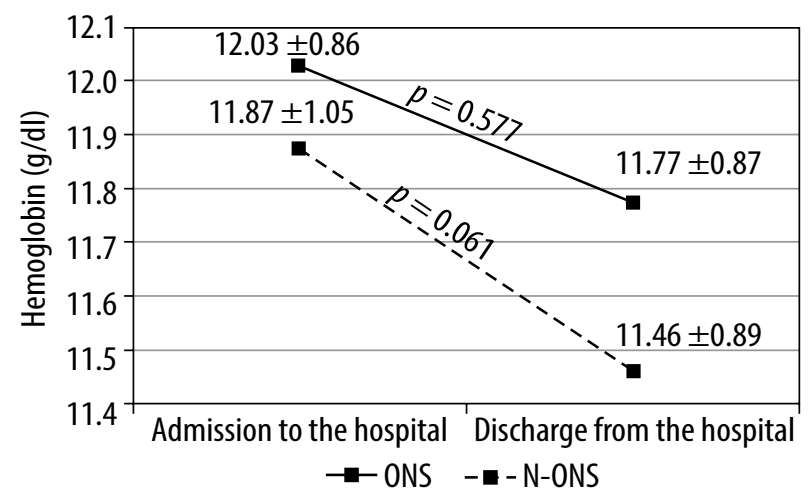

ONS - patients receiving oral nutritional supplements, N-ONS - patients not receiving oral nutritional supplements

FIGURE 2. Change in the haemoglobin value during hospitalisation in the groups of children receiving and not receiving oral nutritional support

mined at discharge from the surgery department (Table 4) were observed, although, contrary to the ONS group $(p=0.577)$, the N-ONS patients demonstrated a clear downward trend of haemoglobin values during hospitalisation, oscillating at the edge of statistical significance $(p=0.061)$ (Fig. 2). No significant differences in the trends of albumin and haematocrit value changes in both populations during hospitalisation were revealed.

\section{DISCUSSION}

An adequate nutritional procedure from the moment of occurrence of a thermal injury to the end of hospitalisation is one of the key components of burn management. This results from induction of post-traumatic hypermetabolic response, which entails a significant increase of energy intake - in particular in the period of prevalence of catabolic processes. Extensiveness of the burn wound seems to be the key element enabling determination of energy intake of a child after thermal injury, provided that, when transferring autologous intermediate thickness grafts, both the body surface damaged in effect of injury and the size of the donor area (surgical injury) are taken into account. While planning an adequate nutritional procedure, it is important to consider the increased protein demand $-3 \mathrm{~g} / \mathrm{kg}$ b.w./24 $\mathrm{h}$ - in addition to adequate energy intake. Adequate supplementation of micro-nutrients and vitamins should also be taken into account. At present, the effect of glutamine on increased survival rate and reduced treatment length (so-called immunomodulating nutrition) has been questioned. At the same time, it is necessary to assess the quantity of glucose administered with the diet, which should supply up to $55 \%$ of the total energy intake. In addition, blood glucose concentration in children with burn injuries should also be controlled and maintained below $8 \mathrm{mmol} / \mathrm{l}$. Excessively high glucose concentrations, which were not observed in the studied population, promote catabolic reactions and increase the risk of infection. According to LeCompte et al., maintaining normal glucose levels can be achieved with propranolol. In hyperglycaemia it is usually administered in dosage of $2 \mathrm{mg} / \mathrm{kg}$ b.w. daily in divided doses [1-5].

Many researchers emphasise the importance of early nutritional intervention and possibly early introduction of oral feeding in children with burn injuries. Planning and implementation of an adequate nutritional procedure should be preceded with metabolic evaluation of the patient and determination of laboratory nutritional status indicators at admission to hospital, which is highlighted by the authors of this publication. According to Chan and Chan, immediate introduction of an adequate nutritional intervention, regardless of administration route (enteral vs. parenteral), reduces the risk of early and late complications, probability of infection, and long-term effects of thermal injury. Khorasani and Mansouri demonstrate that early introduction of enteral feeding reduces the length of hospital stay and decreases the mortality rate $[6,7]$.

The selection of an appropriate route of nutritional intervention depends on the extensiveness and location of thermal injury and progression of burn disease. Oral access is preferred, although in certain cases it is necessary to introduce enteral nutrition or even temporary total parenteral nutrition (TPN). Dylewski et al. state that TPN is a safe and effective alternative to enteral nutrition. The authors of this publication agree with this statement and emphasise the lack of complications when selecting this procedure in the population under study. On the other hand, Suri et al. believe that an optimal, early nutritional intervention can be implemented with the use of a nasogastric tube, while Kreis et al. encourage use of percutaneous endoscopic gastrostomy (PEG) in children with burn injuries requiring long-term nutritional management. According to the authors of this publication, such treatment seems to be reserved only for a small percentage of patients whose period of hospitalisation is prolonged [8-10].

An adequate nutritional procedure depends on the age of the patients and the occurrence, in particular in younger children, of a more severe course of burn disease and therefore an increased risk of nutritional complications. Inhibited intake of an ordered high-energy diet 
rich in proteins is also significantly affected by supply of hospital meals and fixed hours of their consumption. Therefore, in order to ensure adequate caloric intake, all patients in the study required additional food provided by their parents. Such additional feeding cannot be considered a balanced diet. It enables adequate energy intake but in most cases fails to supply the required quantity of proteins, and macro- and micronutrients. In such cases, introduction of ONS, which - in the studied material was strictly required by nearly a half of the children with burn injuries, seems to be crucial. ONS ensures both adequate caloric supply and enables proper balancing of the diet. All the above affect the general condition of a child after thermal injury (better nutrition at discharge) and better wound healing rate. Tolerance to flavour seems to be the only obstacle in applying ONS in children above one year of age with burns [3].

The effects of thermal injury go beyond the length of wound healing or burn disease. Both the induction of post-traumatic hypermetabolic response and significant homeostatic disturbances result in frequent late effects of burns in children. Gottschlich et al. point out chronic low blood concentrations of vitamin D in patients with burns and the necessary of vitamin supplementation. Chao et al. describe long-term disturbances in muscle protein exchange related to a negative net protein balance event in the post-meal periods, which promotes cachectic processes. This phenomenon is most frequently observed in the first year after thermal injury. This imposes regular follow-ups with consideration of full metabolic evaluation and proper development of children treated for burns. This procedure will enable detection of any potential abnormalities and introduction of adequate treatment to prevent occurrence or progression of late effects of burns. According to the authors of this publication, a complete nutritional evaluation and proper development control should be performed at least twice - one year and two years after completion of the burn wound healing process $[11,12]$.

\section{CONCLUSIONS}

An adequate nutritional procedure is one of the key elements of treating a child after thermal injury. Implementation of the adequate nutritional intervention affects both the course of burn disease management and the wound healing rate.

Oral nutritional supplements play an increasing role in the treatment of paediatric patients with burn injuries. Administration of ONS has a significant impact on the nutritional status of burnt children at discharge from hospital and accelerates the wound healing process.

\section{REFERENCES}

1. Coss-Bu J, Hamilton-Reeves J, Patel J, et al. Protein requirements of the critically ill pediatric patient. Nutr Clin Pract 2017; 32 (1 Suppl): 128S-141S.

2. LeCompte MT, Rae L, Kahn SA. A survey of the use of propranolol in burn centers: Who, what, when, why. Burns 2017; 43: 121-126.

3. Rousseau AF, Losser MR, Ichai C, Berger MM. ESPEN endorsed recommendations: nutritional therapy in major burns. Clin Nutr 2013; 32: 497-502.

4. Vijfhuize S, Verburg M, Marino L, et al. An evaluation of nutritional practice in a paediatric burns unit. S Afr Med J 2010; 100: 383-386.

5. Williams GJ, Herndon DN. Modulating the hypermetabolic response to burn injuries. J Wound Care 2002; 11: 87-89.

6. Chan MM, Chan GM. Nutritional therapy for burns in children and adults. Nutrition 2009; 25: 261-269.

7. Khorasani EN, Mansouri F. Effect of early enteral nutrition on morbidity and mortality in children with burns. Burns 2017; 36: 1067-1071.

8. Dylewski ML, Baker M, Prelack K, et al. The safety and efficacy of parenteral nutrition among pediatric patients with burn injuries. Pediatr Crit Care Med 2013; 14: 120-125.

9. Kreis BE, Middelkoop E, Vloemans AF, Kreis RW. The use of a PEG tube in a burn centre. Burns 2002; 28: 191-197.

10. Suri MP, Dhingra VJ, Raibagkar, SC, Mehta DR. Nutrition in burns: need for an aggressive dynamic approach. Burns 2006; 32: 880-884.

11. Chao T, Herndon DN, Porter C, et al. Skeletal muscle protein breakdown remains elevated in pediatric burn survivors up to one-year post-injury. Shock 2015; 44: 397-401.

12. Gottschlich MM, Mayes T, Khoury J, Kagan RJ. Clinical trial of vitamin D2 vs D3 supplementation in critically ill pediatric burn patients. J Parenter Enteral Nutr 2017; 41: 412-421.

\section{DISCLOSURE}

The authors declare no conflict of interest. 